\title{
ANÁLISE QUÍMICA DA MADEIRA COM CASCA EM SISTEMAS FLORESTAIS DE CURTA ROTAÇÃO'
}

\author{
Éder Aparecido Garcia ${ }^{2 *}$, Saulo Philipe Sebastião Guerra ${ }^{3}$, Humberto de Jesus Eufrade Junior ${ }^{4}$, Claudio \\ Angeli Sansígolo ${ }^{5}$, Kléber Pereira Lanças ${ }^{6}$ e Fábio Minoru Yamaji
}

\begin{abstract}
${ }^{1}$ Recebido em 09.01.2014 aceito para publicação em 11.11.2015.
${ }^{2}$ Universidade Estadual Paulista Júlio de Mesquita Filho, Doutorado em Ciências Florestais, Departamento de Ciência Florestal, Faculdade de Ciências Agronômicas, Botucatu, SP - Brasil. E-mail: <garcia.florestal@gmail.com>.

${ }^{3}$ Universidade Estadual Paulista Júlio de Mesquita Filho, Departamento de Economia, Sociologia e Tecnologia, Faculdade de Ciências Agronômicas, Botucatu, SP - Brasil. E-mail: <ssguerra@fca.unesp.br>.

${ }^{4}$ Universidade Estadual Paulista Júlio de Mesquita Filho, Programa de Pós-Graduação em Ciência Florestal, Departamento de Ciência Florestal, Faculdade de Ciências Agronômicas, Botucatu, SP - Brasil. E-mail: <hdjejunior@gmail.com>.

${ }^{5}$ Universidade Estadual Paulista Júlio de Mesquita Filho, Departamento de Ciência Florestal, Faculdade de Ciências Agronômicas, Botucatu, SP - Brasil. E-mail: <sansigolo@fca.unesp.br>.

${ }^{6}$ Universidade Estadual Paulista Júlio de Mesquita Filho, Departamento de Engenharia Rural, Faculdade de Ciências Agronômicas, Botucatu, SP - Brasil. E-mail: <kplancas@fca.unesp.br>.

${ }^{7}$ Universidade Federal de São Carlos, Departamento de Ciências Ambientais, Sorocaba, SP - Brasil. E-mail: < fmyamaji@ufscar.br>.

${ }^{*}$ Autor para correspondência.
\end{abstract}

RESUMO - Os sistemas florestais de curta rotação possuem elevada produtividade em reduzido espaço de tempo e área, entretanto poucos trabalhos enfocam a qualidade da biomassa florestal produzida nessas condições. De fato, a qualidade da matéria-prima é muito importante para alto rendimento nos sistemas energéticos. Dessa forma, este trabalho teve como objetivo estudar a madeira com casca proveniente de diferentes sistemas florestais adensados, por meio do conhecimento do poder calorífico superior e dos principais componentes químicos da madeira - lignina insolúvel, extrativos e cinzas. Adicionalmente, estudou-se o acúmulo desses componentes por árvore ao longo do tempo. As florestas clonais de Eucalyptus urophylla $\mathrm{x}$ Eucalyptus grandis foram plantadas em cinco espaçamentos de plantio e três níveis de adubação. Aos 18, 24 e 30 meses após o plantio, coletaram-se árvores-amostra, das quais foram separados discos da região do DAP para análises químicas. No geral, os teores de lignina insolúvel, extrativos totais e cinzas obtidos nos diferentes tratamentos estão de acordo com os encontrados na literatura e assemelham-se aos das árvores mais velhas da mesma espécie. $\mathrm{O}$ acúmulo dos componentes químicos por árvore foi maior em florestas menos adensadas (espaçamentos mais amplos).

Palavras-chave: Madeira com casca; Eucalyptus sp.; Energia florestal.

\section{CHEMICAL ANALYSIS OF WOOD WITH BARK IN SHORT ROTATION FORESTRY SYSTEMS}

\begin{abstract}
The short rotation forestry systems have high productivity in short period of time and area, however, few studies focused on the quality of forest biomass produced in these conditions. In fact, the quality of the raw material is very important for high performance of energy systems. Thus, the present work aimed to study the wood with bark from different dense forest systems, through knowledge of the gross calorific value and the main chemical components of wood-insoluble lignin, extractives and ash. Additionally, we studied the accumulation of these components in the tree over time. Clonal forestry of Eucalyptus urophylla $x$ Eucalyptus grandis were planted in five planting spaces and three fertilization levels. At 18, 24 and 30 months after planting, sample trees was collected and disks from the DBH region were separated for the chemical analyses. Overall, the levels of insoluble lignin, extractives and ash obtained from the different treatments
\end{abstract}


are in agreement with the literature and the values are similar with other older trees from the same species. The accumulation of chemical components in each tree was higher in less dense forests (more space between trees).

Keywords: Wood with bark; Eucalyptus sp; Forest energy.

\section{INTRODUÇÃO}

A produção de biomassa para energia tem sido realizada há muito tempo no Brasil para abastecer sistemas industriais, como as termelétricas e fábricas que possuem caldeiras. O sistema de produção nas florestas energéticas prima pela produção de biomassa em menor área e com ciclos de corte menores. Os sistemas florestais de curta rotação, short rotations, já têm sido praticados em outros países, como na Itália. No entanto, algumas características químicas da madeira têm que ser consideradas para uso energético, como o poder calorífico, os teores de lignina, extrativos totais e cinzas.

Sua madeira é constituída pelos componentes inorgânicos como os extrativos e as cinzas e por substâncias macromoleculares, que são os polissacarídeos (celulose e hemicelulose) e a lignina. As macromoléculas de lignina são formadas de maneira diferente dos polissacarídeos, pois possuem um sistema aromático composto de unidades de fenilpropano. Durante o desenvolvimento das células, a lignina é incorporada como o último componente na parede celular, interpenetrando as fibrilas. O teor de extrativos nas folhosas fica entre $1 \%$ e $5 \%$ e o teor de lignina, entre 16 e $24 \%$ (KLOCK et al., 2005).

A relação entre os componentes químicos é fundamental na definição do processo de aproveitamento da biomassa, pois as diferentes frações de componentes e suas abundâncias relativas denotam seus potenciais distintos de uso (CASSELLA, 2012). Para a geração de energia, alguns dos extrativos da madeira contribuem para aumentar o poder calorífico (PHILIPP; D'ALMEIDA, 1988).

O poder calorífico superior (PCS) de resíduos vegetais varia de 4.500 a $5.000 \mathrm{kcal} / \mathrm{kg}$ (PINHEIRO et al., 2005). Em seu trabalho, Costa (2006), estudando clones híbridos de Eucalyptus grandis x Eucalyptus urophylla, obteve poder calorífico superior do lenho ou da casca com valor médio de $4.673 \mathrm{kcal} / \mathrm{kg}$.

Trabalhos recentes têm evidenciado que as espécies e os sítios de produção também interferem na qualidade da madeira para bioenergia. Neves et al. (2011) avaliaram os teores de extrativos totais, lignina e cinzas na madeira dos clones de Eucalyptus sp I144, I220 e 3334, todos com idade de 68 meses, no espaçamento de $3,5 \mathrm{~m} \mathrm{x}$ $2,5 \mathrm{~m}$, em dois locais de produção distintos. Esses autores encontraram teores de 1,89 a $4,12 \%$ de extrativos totais, 27,86 a $31,31 \%$ de lignina e 0,15 a $0,25 \%$ de cinzas.

Avaliando a madeira de Eucalyptus grandis aos 5 anos de idade, com a aplicação de biossólidos e de fertilizantes químicos convencionais, Barreiros et al. (2007) constataram que o teor médio de cinzas foi de $0,26 \%$ e aumentou significativamente com o incremento da dose de aplicação de biossólido.

A madeira de Eucalyptus grandis de quatro idades, $10,14,20$ e 25 anos, e três posições no sentido basetopo foi avaliada por Silva et al. (2005), que encontraram valor médio de extrativos totais de $4,08 \%$ e $27,22 \%$ para lignina. Esses autores constataram a evidência de que o teor de extrativos em árvores mais jovens tende a ser menor que em árvore mais velhas e a composição química da madeira variou mais no sentido longitudinal das árvores do que no decorrer do tempo.

Este trabalho teve como objetivo avaliar a madeira com casca proveniente de sistemas florestais de curta rotação, por meio da determinação do poder calorífico e dos componentes químicos - lignina, extrativos totais e cinzas - que atuam durante o processo energético. Adicionalmente, como nova proposta de trabalho, foi realizada uma abordagem sobre o acúmulo desses compostos na madeira, contribuindo, assim, para futuras pesquisas na área de biomassa florestal.

\section{MATERIAL E MÉTODOS}

\section{1. Área experimental}

O experimento foi realizado na Fazenda Três Sinos, localizada na Rodovia Gastão dal Farra, km 5, no Município de Botucatu, Estado de São Paulo. Para este estudo foi utilizado o clone C219 - híbrido de Eucalyptus urophylla x Eucalyptus grandis. O experimento foi realizado em 5,8 ha com solo predominante do tipo Latossolo Vermelho-Amarelo e textura média. A área 
experimental está localizada numa altitude aproximada de 875 m e situa-se entre as longitudes de $48^{\circ} 24^{\prime} 43^{\prime \prime}$ $\mathrm{W}$ e $48^{\circ} 26^{\prime} 21^{\prime \prime} \mathrm{W}$ e entre as latitudes de $22^{\circ} 58^{\prime} 10^{\prime \prime} \mathrm{S}$ e 225' $25^{\prime \prime} \mathrm{S}$. Essas coordenadas foram obtidas através dos mapas da região disponível no Departamento de Engenharia Rural da FCA/UNESP. O clima predominante no município, segundo os critérios adotados por Koppen, é Cfa, clima mesotérmico, com temperaturas médias superiores a $10^{\circ} \mathrm{C}$, cuja temperatura do mês mais quente é igual ou superior a $22^{\circ} \mathrm{C}$, estando o índice pluviométrico anual em torno de $1.516 \mathrm{~mm}$.

\subsection{Tratamentos}

Os tratamentos foram implantados em função dos espaçamentos de plantio e das doses de adubação. Os espaçamentos adotados foram de $2,8 \times 0,5 \mathrm{~m} ; 2,8$ x 1,0 m; 2,8 x1,5 m;2,8 x 2,0 m; e 2,8 x 2,5 m. Cada um desses espaçamentos recebeu três doses de adubação, sendo a dose 1 constituída de 70 g/planta de NPK 630-6 no plantio e adubação de cobertura de $110 \mathrm{~g} /$ planta de NPK 20-0-20 com B e Zn parcelada ao longo do primeiro ano do experimento, sendo a dose 2 o dobro da dose 1 e a dose 3 , quatro vezes a dose 1 . O delineamento experimental foi inteiramente casualizado, organizado em esquema fatorial: cinco espaçamentos, três níveis de adubação e três idades de colheita (18,24 e 30 meses). Os dados de acúmulo dos componentes químicos na biomassa foram analisados no pacote computacional Statistica, da empresa Statsoft ${ }^{\mathbb{B}}$ South America (2013).

\subsection{Coleta das árvores-amostra}

Para este trabalho foram mensuradas 60 árvores de cada tratamento. Excluindo-se a região periférica do povoamento florestal, foram mensurados os diâmetros à altura do peito (DAP) para cálculo do diâmetro quadrático médio - parâmetro utilizado como critério para seleção das árvores abatidas para o estudo.

Dessa forma, foram abatidas quatro árvores-amostra por tratamento aos 18, 24 e 30 meses de idade para a realização da cubagem rigorosa e utilização nas avaliações de densidade básica da madeira, biomassa com casca e análises químicas.

\subsection{Densidade básica da madeira e a biomassa}

Os discos de madeira com casca foram coletados ao longo do fuste das árvores nas posições $0 \%$, DAP, $25 \%, 50 \%, 75 \%$ e $100 \%$ da altura total (PEREIRA et al., 1986; PEREIRA et al., 2002; VALE et al., 2000; GOULART et al., 2003), e todos eles tinham cerca de $5 \mathrm{~cm}$ de espessura, exceto aqueles coletados na região do topo do fuste, que possuíam, no mínimo, $3 \mathrm{~cm}$ de diâmetro.

A metade de cada disco foi retirada para determinar a densidade básica da madeira com casca, seguindose o método do máximo teor de umidade, cujo modelo matemático considera a massa saturada e a massa seca e consiste no seguinte procedimento (VITAL, 1984): as amostras foram retiradas dos discos e colocadas em recipiente com água, permanecendo por cerca de 20 dias (COSTA, 2006); as amostras saturadas foram colocadas em bancadas e o excesso de água, retirado com papel-toalha; a massa saturada foi determinada e, em seguida, as amostras foram secas ao ar e depois colocadas em estufa a $103 \pm 2{ }^{\circ} \mathrm{C}$ até atingirem massa constante.

A biomassa da madeira com casca foi calculada pelo produto resultante dos valores de volume obtido na cubagem rigorosa e da densidade básica média da madeira com casca de cada árvore-amostra.

Análises químicas e o cálculo do acúmulo dos componentes

Para determinação do poder calorífico superior (PCS) e componentes químicos - lignina, extrativos totais e cinzas - foi utilizada uma amostra composta de quatro árvores representativas por tratamento.

A preparação das amostras compostas para análises químicas seguiu-se a norma TAPPI T264 om-88. Foi utilizada a metade de cada disco de madeira com casca da região do DAP das quatro árvores selecionadas por tratamento. Os discos foram cortados e moídos em moinho tipo Willey e, depois, misturados para obtenção de uma amostra homogênea.

O PCS foi determinado pelo método da bomba calorimétrica de acordo com a NBR 8633 (ABNT, 1984). O ensaio foi realizado no Laboratório de Física Aplicada do IBB/UNESP, Botucatu, SP.

Os componentes químicos da madeira com casca foram determinados de acordo com as normas TAPPI T222 om-88 para análise de extrativos totais e TAPPI T222 cm-83 para análise de lignina insolúvel. O teor de cinzas foi calculado após as amostras ficarem na mufla a uma temperatura de $575^{\circ} \mathrm{C}$, durante $3 \mathrm{~h}$. Ambas as análises foram realizadas no Laboratório de Análises Químicas, Departamento de Ciências Florestais, FCA/ UNESP, Botucatu, SP.

Revista Árvore, Viçosa-MG, v.40, n.1, p.163-171, 2016

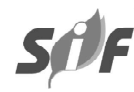


Com os teores médios dos componentes químicos da madeira, foi calculado o acúmulo por árvore, em kg.árvore ${ }^{-1}$, por meio do produto entre teor obtido e a biomassa de madeira com casca das árvores.

\section{RESULTADOS}

De acordo com as Tabelas 1, 2 e 3, percebeu-se que o teor de extrativos para todos os espaçamentos e adubações, na idade de 18 meses, foi de $2,61 \%$, passando para $3,20 \%$ aos 24 meses e $3,58 \%$ aos 30 meses. No caso do teor de lignina, aos 18 meses foi encontrada uma média geral de $22,25 \%$, passando para $22,86 \%$ aos 24 meses e $21,68 \%$ aos 30 meses. Já para os teores de cinzas foi percebido que o teor era de $1 \%$ aos 18 meses, $0,99 \%$ aos 24 meses e $0,74 \%$ aos 30 meses.

Tabela 1 - Teores de cinzas na madeira com casca de Eucalyptus urophylla x Eucalyptus grandis aos 18, 24 e 30 meses após o plantio. FCA/UNESP, Botucatu, SP, 2013.

Table 1-Ash content in wood with bark of Eucalyptus urophylla $x$ Eucalyptus grandis at 18, 24 and 30 months after planting. FCA/UNESP, Botucatu, SP, Brazil, 2013.

\begin{tabular}{|c|c|c|c|c|}
\hline \multirow[t]{2}{*}{ Espaçamento (m) } & \multirow[t]{2}{*}{ Nível de adubação } & \multicolumn{3}{|c|}{ Idade (meses) } \\
\hline & & 18 & 24 & 30 \\
\hline \multirow{3}{*}{$2,8 \times 0,5$} & 1 & 0,80 & 1,00 & 0,88 \\
\hline & 2 & 0,96 & 0,87 & 0,95 \\
\hline & 3 & 0,94 & 1,03 & 0,87 \\
\hline \multirow{3}{*}{$2,8 \times 1,0$} & 1 & 0,86 & 0,96 & 0,66 \\
\hline & 2 & 0,86 & 0,95 & 0,86 \\
\hline & 3 & 1,04 & 0,99 & 1,00 \\
\hline \multirow{3}{*}{$2,8 \times 1,5$} & 1 & 1,00 & 0,88 & 0,87 \\
\hline & 2 & 1,05 & 1,14 & 0,40 \\
\hline & 3 & 1,05 & 0,99 & 0,80 \\
\hline \multirow{3}{*}{$2,8 \times 2,0$} & 1 & 0,96 & 0,95 & 0,57 \\
\hline & 2 & 1,19 & 0,88 & 0,52 \\
\hline & 3 & 1,05 & 1,01 & 0,88 \\
\hline \multirow{3}{*}{$2,8 \times 2,5$} & 1 & 0,95 & 1,13 & 0,67 \\
\hline & 2 & 1,30 & 0,96 & 0,64 \\
\hline & 3 & 1,06 & 1,08 & 0,55 \\
\hline
\end{tabular}

Tabela 2 - Teor de extrativos totais na madeira com casca de Eucalyptus urophylla x Eucalyptus grandis aos $18,24 \mathrm{e}$ 30 meses após o plantio. FCA/UNESP, Botucatu, SP, 2013.

Table 2 - Content of total extractives in wood with bark of Eucalyptus urophylla $x$ Eucalyptus grandis at 18,24 and 30 months after planting. FCA/UNESP, Botucatu, SP, Brazil, 2013.

\begin{tabular}{llllr}
\hline Espaçamento $(\mathrm{m})$ & Nível de adubação & \multicolumn{2}{c}{ Idade (meses) } \\
\cline { 3 - 5 } & & 18 & 24 & 30 \\
\hline \multirow{2}{*}{$2,8 \times 0,5$} & 1 & 3,15 & 2,68 & 3,12 \\
& 2 & 2,49 & 2,80 & 3,48 \\
& 3 & 3,92 & 3,37 & 2,14 \\
\hline \multirow{2}{*}{$2,8 \times 1,0$} & 1 & 2,07 & 4,10 & 3,33 \\
& 2 & 1,64 & 3,65 & 2,07 \\
& 3 & 1,80 & 3,22 & 3,81 \\
\hline \multirow{2}{*}{$2,8 \times 1,5$} & 1 & 2,06 & 4,63 \\
& 2 & 2,67 & 2,45 & 3,48 \\
$2,8 \times 2,0$ & 3 & 3,12 & 3,64 & 3,36 \\
\hline \multirow{2}{*}{$2,8 \times 2,0$} & 1 & 2,76 & 3,26 & 2,53 \\
& 2 & 3,22 & 4,29 & 3,93 \\
& 3 & 2,19 & 2,69 & 3,02 \\
\hline
\end{tabular}

Revista Árvore, Viçosa-MG, v.40, n.1, p.163-171, 2016 
Tabela 3 - Teor de lignina na madeira com casca de Eucalyptus urophylla x Eucalyptus grandis aos 18, 24 e 30 meses após o plantio. FCA/UNESP, Botucatu, SP, 2013.

Table 3 - Lignin in wood with bark of Eucalyptus urophylla $x$ Eucalyptus grandis at 18, 24 and 30 months after planting. FCA/UNESP, Botucatu, SP, Brazil, 2013.

\begin{tabular}{|c|c|c|c|c|}
\hline \multirow[t]{2}{*}{ Espaçamento (m) } & \multirow[t]{2}{*}{ Nível de adubação } & \multicolumn{3}{|c|}{ Idade (meses) } \\
\hline & & 18 & 24 & 30 \\
\hline \multirow{3}{*}{$2,8 \times 0,5$} & 1 & 21,35 & 21,16 & 21,57 \\
\hline & 2 & 22,38 & 21,82 & 23,11 \\
\hline & 3 & 21,25 & 21,92 & 19,22 \\
\hline \multirow{3}{*}{$2,8 \times 1,0$} & 1 & 22,39 & 23,34 & 21,89 \\
\hline & 2 & 22,34 & 22,54 & 22,08 \\
\hline & 3 & 22,33 & 23,11 & 23,02 \\
\hline \multirow{3}{*}{$2,8 \times 1,5$} & 1 & 22,22 & 23,14 & 21,10 \\
\hline & 2 & 22,17 & 22,97 & 19,97 \\
\hline & 3 & 21,29 & 23,38 & 21,26 \\
\hline \multirow{3}{*}{$2,8 \times 2,0$} & 1 & 22,41 & 23,41 & 22,15 \\
\hline & 2 & 23,36 & 22,83 & 22,91 \\
\hline & 3 & 22,96 & 23,64 & 22,37 \\
\hline \multirow{3}{*}{$2,8 \times 2,5$} & 1 & 22,84 & 23,31 & 21,63 \\
\hline & 2 & 22,14 & 23,34 & 22,52 \\
\hline & 3 & 22,37 & 23,03 & 20,33 \\
\hline
\end{tabular}

Considerando-se o acúmulo da biomassa seca de madeira com casca, o fatorial triplo foi significativo, com á $=0,05$, para o acúmulo de extrativos totais e cinzas, enquanto para a lignina acumulada as interações significativas foram idade de colheita $\mathrm{x}$ espaçamento. As demais interações foram significativas a 5\% de significância para os extrativos totais e cinzas.

O maior acúmulo de extrativos totais, considerando a interação espaçamento $\mathrm{x}$ idade de colheita, foi de 2,16 kg.árvore ${ }^{-1}$, no espaçamento 2,8 x 2,5 m, na idade de 30 meses (Tabela 4). Para a quantidade de lignina, o maior acúmulo foi de $9,05 \mathrm{~kg}$.árvore ${ }^{-1}$ no espaçamento $2,8 \times 2,5 \mathrm{~m}$, colhida aos 30 meses. A quantidade de cinzas deve ser minimizada para a utilização da madeira como fonte energética. Neste estudo, observaram-se menores acúmulos de cinza no espaçamento de 2,8 x $0,5 \mathrm{~m}$ aos 18 e 24 meses após o plantio.

Em relação à interação espaçamento x nível de adubação, o maior acúmulo de extrativos totais foi de $1,71 \mathrm{~kg}$.árvore ${ }^{-1}$ no espaçamento de $2,8 \times 2,5 \mathrm{~m}$, na maior adubação (Tabela 4). Já para o teor de lignina os maiores acúmulos ocorreram no espaçamento de 2,8 x 2,5 m, nas adubações 2 e 3 , sendo o nível 2 equivalente à adubação operacional da empresa. Os menores acúmulos de cinzas na madeira ocorreram no espaçamento de $2,8 \times 0,5 \mathrm{~m}$, nas fertilizações 2 e 3 .
Uma das justificativas para o aumento do teor de cinzas na madeira está associada à quantidade de impurezas, provenientes de poeira e de casca, que aumentam a quantidade de silicatos (BRAND, 2010). $\mathrm{Na}$ Tabela 5, são apresentados os valores de porcentagem de casca dos tratamentos testados na pesquisa.

Em relação à energia da madeira, constatou-se que não houve diferença do poder calorífico ao serem considerados os espaçamentos nas idades de 18, 24 e 30 meses. Entretanto, nas condições experimentadas neste trabalho, a madeira com casca nos espaçamentos de 2,8 x 1,0 m; 2,8 x 2,0 m; e 2,8 x 2,5 m, aos 30 meses, apresentaram poder calorífico superior significativamente menor que nas demais idades, nesses espaçamentos.

\section{DISCUSSÃO}

Para aumentar a eficiência energética nos sistemas industriais, é importante conhecer a constituição química da biomassa utilizada no processo, como o teor de extrativos totais, teor de lignina e teor de cinzas.

Estudando a madeira de clones de Eucalyptus ssp, Neves et al. (2011) encontraram teores de cinzas entre $0,15 \%$ e $0,25 \%$ em experimentos no Estado de Minas Gerais, cuja madeira é destinada à produção de carvão vegetal. Esses valores são menores em relação aos teores de cinzas encontrados neste estudo, que

Revista Árvore, Viçosa-MG, v.40, n.1, p.163-171, 2016 
Tabela 4 - Acumulo de extrativos totais, lignina e cinzas na madeira com casca de Eucalyptus urophylla $\mathrm{x}$ Eucalyptus grandis, na interação espaçamento $\mathrm{x}$ idade de colheita e na interação espaçamento x nível de adubação. FCA/UNESP, Botucatu, SP, 2013.

Table 4-Accumulation of extractives, lignin and ash in wood with bark of Eucalyptus urophylla $x$ Eucalyptus grandis in interaction spacing and harvest age and interaction spacing $x$ fertilizer level. FCA/UNESP, Botucatu, SP, Brasil, 2013.

\begin{tabular}{|c|c|c|c|c|c|c|}
\hline \multicolumn{7}{|c|}{ Extrativos totais (kg/árvore) } \\
\hline \multirow{2}{*}{$\begin{array}{l}\text { Espaçamento } \\
\text { (m) }\end{array}$} & \multicolumn{3}{|c|}{ Idade de colheita (meses) } & \multicolumn{3}{|c|}{ Nível de Adubação } \\
\hline & 18 & 24 & 30 & 1 & 2 & 3 \\
\hline $2,8 \times 0,5$ & $0,24 \mathrm{Bb}$ & $0,29 \mathrm{Cb}$ & $0,41 \mathrm{Da}$ & $0,25 \mathrm{Db}$ & $0,29 \mathrm{Cb}$ & $0,40 \mathrm{Ca}$ \\
\hline $2,8 \times 1,0$ & $0,23 \mathrm{Bc}$ & $0,42 \mathrm{Cb}$ & $0,69 \mathrm{Ca}$ & $0,45 \mathrm{Cb}$ & $0,37 \mathrm{Cb}$ & $0,53 \mathrm{Ca}$ \\
\hline $2,8 \times 1,5$ & $0,45 \mathrm{Ab}$ & $0,59 \mathrm{Bb}$ & $1,07 \mathrm{Ba}$ & $0,70 \mathrm{Ba}$ & $0,64 \mathrm{Ba}$ & $0,76 \mathrm{Ba}$ \\
\hline $2,8 \times 2,0$ & $0,51 \mathrm{Ac}$ & $0,79 \mathrm{Ab}$ & $1,14 \mathrm{Ba}$ & $0,64 \mathrm{Bb}$ & $1,02 \mathrm{Aa}$ & $0,78 \mathrm{Bb}$ \\
\hline $2,8 \times 2,5$ & $0,51 \mathrm{Ac}$ & $0,74 \mathrm{Ab}$ & $2,16 \mathrm{Aa}$ & $0,92 \mathrm{Ab}$ & $0,87 \mathrm{Ab}$ & $1,71 \mathrm{Aa}$ \\
\hline \multicolumn{7}{|c|}{ Lignina (kg/árvore) } \\
\hline Espaçamento & \multicolumn{3}{|c|}{ Idade de colheita (meses) } & \multicolumn{3}{|c|}{ Nível de Adubação } \\
\hline$(\mathrm{m})$ & 18 & 24 & 30 & 1 & 2 & 3 \\
\hline $2,8 \times 0,5$ & $1,60 \mathrm{Cb}$ & $2,05 \mathrm{Cb}$ & $3,06 \mathrm{Da}$ & $1,75 \mathrm{Db}$ & $2,16 \mathrm{Dab}$ & $2,80 \mathrm{Da}$ \\
\hline $2,8 \times 1,0$ & $2,83 \mathrm{Bb}$ & $2,68 \mathrm{Cb}$ & $5,04 \mathrm{Ca}$ & $3,13 \mathrm{Ca}$ & $3,56 \mathrm{Ca}$ & $3,86 \mathrm{Ca}$ \\
\hline $2,8 \times 1,5$ & $3,74 \mathrm{ABb}$ & $4,50 \mathrm{Bb}$ & $5,86 \mathrm{Ca}$ & $4,52 \mathrm{Ba}$ & $4,66 \mathrm{Ba}$ & $4,92 \mathrm{Ba}$ \\
\hline $2,8 \times 2,0$ & $4,28 \mathrm{Ac}$ & $5,31 \mathrm{ABb}$ & $8,10 \mathrm{Ba}$ & $5,15 \mathrm{ABb}$ & $6,05 \mathrm{Aab}$ & $6,49 \mathrm{Aa}$ \\
\hline $2,8 \times 2,5$ & $4,45 \mathrm{Ac}$ & $5,80 \mathrm{Ab}$ & $9,05 \mathrm{Aa}$ & $5,54 \mathrm{Ab}$ & $6,59 \mathrm{Aa}$ & $7,30 \mathrm{Aa}$ \\
\hline \multicolumn{7}{|c|}{ Cinzas (kg/árvore) } \\
\hline Espaçamento & \multicolumn{3}{|c|}{ Idade de colheita (meses) } & \multicolumn{3}{|c|}{ Nível de Adubação } \\
\hline$(\mathrm{m})$ & 18 & 24 & 30 & 1 & 2 & 3 \\
\hline $2,8 \times 0,5$ & $0,07 \mathrm{Db}$ & $0,09 \mathrm{Cb}$ & $0,13 \mathrm{Ca}$ & $0,07 \mathrm{Db}$ & $0,09 \mathrm{Db}$ & $0,13 \mathrm{Da}$ \\
\hline $2,8 \times 1,0$ & $0,12 \mathrm{Cb}$ & $0,11 \mathrm{Cb}$ & $0,19 \mathrm{Ba}$ & $0,11 \mathrm{Cb}$ & $0,14 \mathrm{Cab}$ & $0,17 \mathrm{Ca}$ \\
\hline $2,8 \times 1,5$ & $0,18 \mathrm{Ba}$ & $0,19 \mathrm{Ba}$ & $0,19 \mathrm{Ba}$ & $0,19 \mathrm{Ba}$ & $0,17 \mathrm{Cb}$ & $0,21 \mathrm{Ba}$ \\
\hline $2,8 \times 2,0$ & $0,20 \mathrm{ABb}$ & $0,22 \mathrm{Bab}$ & $0,24 \mathrm{Aa}$ & $0,18 \mathrm{Bc}$ & $0,21 \mathrm{Bb}$ & $0,27 \mathrm{Aa}$ \\
\hline $2,8 \times 2,5$ & $0,22 \mathrm{Ab}$ & $0,26 \mathrm{Aa}$ & $0,26 \mathrm{Aa}$ & $0,21 \mathrm{Ab}$ & $0,26 \mathrm{Aa}$ & $0,27 \mathrm{Aa}$ \\
\hline
\end{tabular}

ficaram entre $0,40 \%$ e $1,19 \%$. O alto de teor de cinzas encontrado pode ser explicado pela utilização da madeira com casca.

Sansígolo e Ramos (2011) avaliaram a madeira de Eucalyptus grandis aos 4 anos de idade destinada a celulose, em diferentes sítios de produção, encontrando teores médios de extrativos totais entre $1,98 \%$ e 3,31\% e teores médios de lignina entre $22,8 \%$ e $23,8 \%$. Ainda, Silva et al. (2005) encontraram cerca de $23,78 \%$ de lignina em madeira de eucalipto com 10 anos, e Barreiros et al. (2007) estimaram teor de lignina de $24 \%$ em madeira de Eucalyptus grandis, cinco anos após o plantio. Os resultados deste estudo reforçam a ideia de que a madeira no sistema de curta rotação possui valores próximos aos citados na literatura para o sistema convencional de ciclo longo.

Nos processos de conversão da madeira para fins energéticos, os componentes inertes da madeira, as cinzas (Tabela 4), deterioraram os equipamentos. Os teores avaliados por Souza et al. (2009) em espécies florestais da região amazônica ficaram entre $0,27 \%$ e $1,82 \%$ para cinzas e $0,10 \%$ e $0,52 \%$ para a sílica.

Quanto maior a porcentagem de casca, maior o teor de cinzas da biomassa para energia, porque neste componente há presença de impurezas como poeiras, que trazem silicatos do solo. Brand (2010) afirmou que a casca normalmente tem teor de cinzas maior que os outros componentes das árvores, pois participa da proteção do vegetal, sujeita à maior incrustação de material inorgânico. Esse material são poeiras e particulados carreados pelo vento ou, mesmo, pelos processos de colheita e transporte da madeira.

Da madeira com casca produzida neste sistema de curta rotação, observou-se que a quantidade de casca nos fustes era menor em espaçamentos mais adensados. Aos 18 meses de idade, a menor quantidade de casca foi de $13,8 \%$ no espaçamento de $2,8 \times 2,0 \mathrm{~m}$, 
Tabela 5 - Porcentagem de casca no fuste de árvores de Eucalyptus urophylla x Eucalyptus grandis em diferentes espaçamentos, adubações e idades de colheita. FCA/ UNESP, Botucatu, SP, 2013.

Table 5 - Percentage of the bark in the stem of Eucalyptus urophylla $x$ Eucalyptus grandis trees at different spacings, fertilizers and harvest ages. FCA/UNESP, Botucatu, SP, Brazil, 2013.

\begin{tabular}{|c|c|c|c|}
\hline \multicolumn{4}{|c|}{18 meses } \\
\hline \multirow[t]{2}{*}{ Espaçamento (m) } & \multicolumn{3}{|c|}{ Nível de adubação } \\
\hline & 1 & 2 & 3 \\
\hline $2,8 \times 0,5$ & 18,4 & 18,3 & 19,0 \\
\hline $2,8 \times 1,0$ & 18,8 & 17,9 & 19,2 \\
\hline $2,8 \times 1,5$ & 16,6 & 15,4 & 20,3 \\
\hline $2,8 \times 2,0$ & 22,9 & 13,8 & 22,3 \\
\hline $2,8 \times 2,5$ & 16,1 & 18,1 & 14,5 \\
\hline \multicolumn{4}{|c|}{24 meses } \\
\hline \multirow[t]{2}{*}{ Espaçamento (m) } & \multicolumn{3}{|c|}{ Nível de adubação } \\
\hline & 1 & 2 & 3 \\
\hline $2,8 \times 0,5$ & 17,1 & 18,5 & 10,5 \\
\hline $2,8 \times 1,0$ & 17,2 & 19,9 & 18,6 \\
\hline $2,8 \times 1,5$ & 15,7 & 19,1 & 17,2 \\
\hline $2,8 \times 2,0$ & 19,2 & 17,7 & 18,2 \\
\hline $2,8 \times 2,5$ & 19,5 & 18,8 & 16,5 \\
\hline \multicolumn{4}{|c|}{30 meses } \\
\hline \multirow[t]{2}{*}{ Espaçamento (m) } & \multicolumn{3}{|c|}{ Nível de adubação } \\
\hline & 1 & 2 & 3 \\
\hline $2,8 \times 0,5$ & 12,8 & 18,4 & 17,6 \\
\hline $2,8 \times 1,0$ & 17,7 & 18,6 & 18,7 \\
\hline $2,8 \times 1,5$ & 20,4 & 18,7 & 19,2 \\
\hline $2,8 \times 2,0$ & 19,9 & 18,5 & 18,6 \\
\hline $2,8 \times 2,5$ & 18,8 & 16,9 & 16,5 \\
\hline
\end{tabular}

Médias seguidas de mesma letra maiúscula nas colunas não se diferem entre si, ao passo que médias seguidas de mesma letra minúscula, nas linhas, não se diferem pelo teste de Tukey $(\alpha=0,05)$.

na fertilização da empresa, nível 2. Aos 24 meses, a menor quantidade de casca foi de $10,5 \%$, no espaçamento mais adensado. Aos 30 meses, ocorreu tendência semelhante à dos 24 meses, cerca de $12,8 \%$ de casca no espaçamento mais adensado, com menor aplicação de fertilizante. Ressalta-se que, após o processo de carbonização da madeira, as cinzas podem ser reutilizadas nos povoamentos florestais como insumo, haja vista a reconhecida presença de potássio.

Os resultados de PCS neste trabalho, entre 19,25 MJ.kg-1 e 20,15 MJ.kg-1, estão condizentes com os de outras pesquisas da área de biomassa florestal para energia. Para madeira de Eucalyptus benthamii aos 6 anos, Lima et al. (2007) encontraram poder calorífico superior de 4.681 kcal.kg-1 $\left(19,59 \mathrm{MJ} . \mathrm{kg}^{-1}\right)$. Em madeira de Eucalyptus grandis, Vale et al. (2000) verificaram um PCS de 4.641 kcal.kg-1 (19,43 MJ.kg$\left.{ }^{1}\right)$ e Quirino et al. (2005) estabeleceram um intervalo entre 4.501 e $4.790 \mathrm{kcal}^{-\mathrm{kg}^{-1}}$ (18,84 e 20,05 MJ.kg$\left.{ }^{1}\right)$ para a mesma espécie. No mesmo trabalho de Quirino et al. (2005), foi obtido um intervalo de 4.422 e 4.595 kcal. $\mathrm{kg}^{-1}(18,51$ e 19,24 MJ.kg-1) de poder calorífico da madeira de Eucalyptus urophylla.

Para que a madeira seja utilizada como biocombustível, é desejável que ela contenha maiores quantidades de lignina e extrativos, ao passo que as cinzas devem estar em menor quantidade. Este trabalho trouxe nova abordagem sobre o acúmulo desses compostos químicos na árvore, trazendo informações que podem ser relacionadas a outras características da madeira, como o poder calorífico e a produção energética das florestas manejadas no sistema de curta rotação.

\section{CONCLUSÕES}

Os teores dos componentes químicos da madeira com casca de sistemas florestais de curta rotação assemelham-se aos da madeira de povoamentos comerciais em rotações tradicionais.

Revista Árvore, Viçosa-MG, v.40, n.1, p.163-171, 2016 
Os acúmulos de lignina insolúvel, extrativos totais e cinzas variaram em função das diferentes formas de tratamento da floresta. Os maiores acúmulos de lignina, extrativos totais e cinzas na madeira com casca, em kg.árvore ${ }^{-1}$, foram obtidos nos espaçamentos mais amplos.

\section{AGRADECIMENTOS}

Aos professores orientadores, pelos ensinamentos; aos colegas de pós-graduação, pela ajuda nas coletas de dados em campo e em laboratório; e ao Conselho Nacional de Desenvolvimento Científico e Tecnológico (CNPq), pela concessão da Bolsa de Doutorado ao primeiro autor.

\section{REFERÊNCIAS}

\section{ASSOCIAÇÃO BRASILEIRA DE NORMAS TÉCNICA - ABNT. NBR 8633: carvão vegetal - determinação do poder calorífico. Rio de Janeiro: 1984. 12p.}

BARREIROS, R.M.; GONÇALVES, J.L.M.; SANSÍGOLO, C.A.; POGGIANI, F. Modificações na propriedade e nas características físicas e químicas da madeira de Eucalyptus grandis causadas pela adubação com lodo de esgoto tratado. Revista Árvore, v.31, n.1, p.103-111, 2007.

BRAND, M.A. Energia da biomassa florestal. Rio de Janeiro: Interciência, 2010. 131p.

CASSELLA, A. R.; MELLO, L. A.; TEIXEIRA, M. A. G.; ÁVILA, R. A. P. Caracterização de Biomassa por Análise Térmica. In: CONGRESSO BRASILEIRO, 8.; CONGRESSO PANAMERICANO DE ANÁLISE TÉRMICA E CALORIMETRIA, 3., 2012, Campos do Jordão. Anais...São Paulo: Associação Brasileira de Análise Térmica e Calorimetria, 2012.6p.

COSTA, V.E. Caracterização físicoenergética da madeira e produtividade de reflorestamentos de clones de híbridos de Eucalyptus grandis x E.urophylla. 2006. 99f. Tese (Doutorado em Agronomia/Energia na Agricultura) Universidade Estadual Paulista, Faculdade de Ciências Agronômicas, Botucatu, 2006.

GOULART, M.; HASELEIN, C.R.; HOPPE, J.M.; FARIAS, J.A.; PAULESKI, D.T. Massa

Revista Árvore, Viçosa-MG, v.40, n.1, p.163-171, 2016 especifica básica e massa seca de madeira de Eucalyptus grandis sob o efeito do espaçamento de plantio e da posição axial no tronco. Ciência Florestal, v.13, n.2, p.167$175,2003$.

KLOCK, V.; MUÑUZ, G.I.B.; HERNANDEZ, J.A.; ANDRADE, A.S. Química da madeira 3.ed. Manual didático. Curitiba: Universidade Federal do Paraná, 2005. Acessado em 24 maio, 2011. Disponível em: http://marioloureiro.net/ ciencia/biomass/quimicadamadeira.pdf..

LIMA, E.A.; SILVA, H.D.; MAGALHÃES, W.L.E.; LAVORANTI, O.J. Caracterização individual de árvores de Eucalyptus benthamii para uso energético. Colombo: Embrapa Florestas, 2007. (Boletim de Pesquisa e Desenvolvimento, 35)

NEVES, T.A.; PROTÁSIO, T.P.; COUTO, A.M.; TRUGILHO, P.F.; SILVA, V.O.; VIEIRA, C.M.M. Avaliação de clones de Eucalyptus em diferentes locais visando à produção de carvão vegetal. Pesquisa Florestal Brasileira, v.31, p.319330, 2011.

PEREIRA, J.C.D.; HIGA, A.R.; SHIMIZU, J.Y.; HIGA, R.C.V. Comparação da qualidade da madeira de três procedências de Eucalyptus dunnii Maiden, para fins energéticos. Boletim de Pesquisa Florestal, n.13, p.9-16, 1986.

PEREIRA, J.C.D.; LIMA, P.C.F. Comparação da qualidade da madeira de seis espécies de algarobeira para a produção de energia. Boletim de Pesquisa Florestal, n.45, p.99-107, 2002.

PHILIPP, P.; D’ALMEIDA, M.L.O. Celulose e papel: tecnologia de fabricação da pasta celulósica. 2.ed. São Paulo: IPT, 1988.

PINHEIRO, G.F.; RENDEIRO, G.; PINHO, J.T. Densidade energética de resíduos vegetais. Biomassa \& Energia, v.2, n.2, p.113-123, 2005.

QUIRINO, W.F.; VALE, A.T.; ANDRADE, A.P.A.; ABREU, V.L.S.; AZEVEDO, A.C.S. Poder calorífico da madeira e de materiais ligno-celulósicos.

Revista da Madeira, n.89, p.100-106, 2005.

SANSÍGOLO, C.A.; RAMOS, E.S. Quality of wood and pulp from a clone of Eucalyptus grandis 
planted at three locations. Revista Cerne, v.17, n.1, p.47-60, 2011.

SILVA, J.C.; MATOS, J.L.M.; OLIVEIRA, J.T.S.; EVANGELISTA, W.V. Influencia da idade e da posição ao longo do tronco na composição química da madeira de Eucalyptus grandis Hill ex Maiden. Revista Árvore, v.29, n.3, p.455-460, 2005.

SOUZA, S.S.; SANATOS, P.O.; VAREJÃO, M.J.C.; NASCIMENTO, C.C. Detecção do teor de cinzas e sílica em materiais lignocelulósicos. In: REUNIÃO ANUAL DA SBPC, 61., Manaus, 2009. Manaus: UFAM, 2009. Acessado em: 13 out. 2012. Disponível: www.sbpc.net.org.br/livro/61ra/ resuumos/resumos/5044.htm. .

STATSOFT. Statistica 8. Demonstrativo. 2013.

TAPPI - TECHNICALASSOCIATION OF THE PULP AND PAPER INDUSTRY. TAPPI test methods T 204 om-88: solvente extractives of wood and pulp. Atlanta: Tappi Technology Park, 1996.

TAPPI - TECHNICAL ASSOCIATION OF THE PULP AND PAPER INDUSTRY. TAPPI test methods T 222 om-88: acid insoluble lignin in wood and pulp. Atlanta: Tappi Technology Park, 1996.

TAPPI - TECHNICAL ASSOCIATION OF THE PULP AND PAPER INDUSTRY. TAPPI test methods T 264 om-88: preparation of wood for chemical analysis. Atlanta: Tappi Technology Park, 1996.

VALE, A.T.; BRASIL, M.A.M.; CARVALHO, C.M.; VEIGA, R.A.A. Produção de energia do fuste de Eucalyptus grandis e Acacia mangium em diferentes níveis de adubação. Revista Cerne, v.6, n. 1, p.83-88, 2000.

VITAL, B.R. Métodos de determinação da densidade da madeira. Viçosa: SIF, 1984. 21p. (Boletim Técnico, 2). 